\title{
Terman-Merril Application for Intelligence Measurement
}

\author{
Alicia Valdez ${ }^{1}$, Griselda Cortes $^{2}$, Laura Vazquez ${ }^{3}$, \\ Andrea de la Pena ${ }^{4}$ \\ Research Center \\ Autonomous University of Coahuila \\ Coahuila, Mexico
}

\author{
Blanca Montano 5 \\ Human Resources Department \\ Faculty of Accounting and Administration \\ Autonomous University of Coahuila \\ Coahuila, Mexico
}

\begin{abstract}
The computational applications support different processes in organizations, one of these processes are those related to human resources, where one of the activities is the hiring of new personnel; the evaluation of human talent to be integrated into a company can be measured through different tests, one of which is the Terman-Merril intelligence test, which measures the intellectual quotient of candidates with a series of sub-tests. In this project, the waterfall method has been used for the development of a web computational application for the Terman-Merril intelligence test, as well as the management of the users and the results obtained to be visualized in spreadsheets for its subsequent analysis and graphing. The ASP.Net programming language and the SQL Server 2014 database have been used for the programming and the storage of information. As a result, it has been applied successfully in some companies, obtaining measurable and evaluable results on the candidates. The application has also been installed in a computer lab for students enrolled in the Bachelor of Human Resources at Faculty of Accounting and Administration at Coahuila, Mexico.
\end{abstract}

Keywords-Human resources; Terman-Merril test; intelligence test; ASP.Net

\section{INTRODUCTION}

The intelligence research is an important area of knowledge for researchers and practicing psychologists, organizations require tests to evaluate new staff and select those that get the best scores, testing in the selection process is a significant element that has aim to select the high quality personnel in the organization. Special features in the selection process are represented through psychological tests and intelligence tests [1].

The assessment of intelligence required good measurement instruments, consists of standardized questions and tools for assessing an individual potential [2].

At the present day there are various psychometric tools for intelligence measurement, one of these is the "Standard Progressive Matrices Test" (SPMT), which was developed by J.C. Raven (1939) and distributed by US Psychological Corporation, consists of abstract reasoning; puzzle solving, problem solving, learning and patterns recognized [3].

Alternative tools for measuring intellectual abilities were proposed by M.A. Kholodnaya as a development of an onthological approach to intelligence studies, these tests aim to measure categorical and conceptual abilities that underlie intellectual productivity, implies the necessity to derive a new conceptual knowledge by combining three words from completely different semantic contexts into one meaningful sentence [4].

The Terman-Merril Test (TMT) was created in 1960, by L. Terman and M. A. Merril, based on the research work of A. Binet, who was a teacher at Stanford University; for the evaluation of high intellectual capacities, where intelligence is measured as a general capacity starting from a chronological age, obtaining intellectual quotient (IQ) values significant and progressive higher than those obtained with the currently used factor scales, whose primary objective is to determine the intellectual quotient of people.

It is a set of ten structured tests, requires a maximum execution to obtain the best performance of the examinee.

The objectives of the test measure are: Common sense to appreciate social situations, develop the ability to understand concepts expressed in words, knowledge of language, obtain ability to summarize, relate and abstract essential ideas, develop the ability to concentrate and work under pressure, learn to anticipate situations to foresee the future and mentally imagine the solution to a problem. Each series has a time limit and the total test requires 27 minutes, and can be administered individually or collectively [5], [6].

In this project was developed a computational application based in the TMT for intelligence measurement, considering all the variables of the ten tests that integrate it.

The software was used as a tool in a computer laboratory for the human resources students at the Faculty of Accounting and Administration at Autonomous University of Coahuila, else for examining people to be hired by the companies.

The program was created using Active Server Pages (ASP).Net [7] programming language and the SQL Server 2014 database for data storage [8].

\section{Basically, this study has four sections.}

In Section I, the introduction was shown. In Section II, the fundamental concepts are described. Also, the Terman Merril structure was stated. Thus, Section III describes the methodology, and Section IV describes the principal findings of the project. 


\section{FUNDAMENTAL CONCEPTS}

\section{A. Terman-Merril Structure}

It is composed of ten sets of tests that measure different cognitive skills and abilities of people, each of which has a measurement in minutes to be applied, in total the full test can be achieved in 27 minutes. The particular characteristics are described below, the tests are passed when the subject meets the criterion of step for each of them; minimum performance sufficient for the proposed task, being the result dichotomous (overcome / not exceeded). Tests are assessed and scored at more than one mental age level with demanding criteria. The IQ is obtained from the corresponding norms, which relate the chronological age at the time of performing the test with the mental age obtained. As the chronological age increases, the mean and dispersion of the mental ages is also increasing [9].

- Information or knowledge, measure culture and general knowledge, long-term memory, answer in a time limit 2 minutes.

- Understanding, measures the understanding and management of reality.

- Verbal meanings, measures the ability to analyze and synthesize concepts.

- Logical selection, measures the ability to deduce and logically abstract concepts. 2 minutes time.

- Arithmetic, measures reasoning and quantitative concepts. It is answered within a 3 minutes time limit.

- Practical judgment, measure common sense. It is answered in a time limit of 5 minutes.

- Analogies, ability to reason, abstract, generalize and think in an organized way.

- Sentence ordering, planning, organization and understanding of concepts. Attention to detail. Maximum response time 3 minutes.

- Classification, measures the logical discrimination of concepts.

- Seriation, measures the deduction capacity. It is answered in a maximum of 4 minutes. Table I summarizes of test series, skill and time.

TABLE I. TERMAN-MERRIL TEST SERIES

\begin{tabular}{|l|l|l|}
\hline Serie & Skill & Time \\
\hline I & Information & 2 minutes \\
\hline II & Understanding & 2 minutes \\
\hline III & $\begin{array}{l}\text { Verbal } \\
\text { meanings }\end{array}$ & 2 minutes \\
\hline IV & $\begin{array}{l}\text { Logical } \\
\text { selection }\end{array}$ & 3 minutes \\
\hline V & Arithmetic & 5 minutes \\
\hline VI & $\begin{array}{l}\text { Practical } \\
\text { judgment }\end{array}$ & 2 minutes \\
\hline VII & Analogies & 2 minutes \\
\hline VIII & $\begin{array}{l}\text { Sentence } \\
\text { ordering }\end{array}$ & 3 minutes \\
\hline IX & Classification & 2 minutes \\
\hline X & Seriation & 4 minutes \\
\hline Total & & 27 minutes \\
\hline & &
\end{tabular}

Once the methodology comprising the TM intelligence test is understood, the software development component is carried out.

\section{B. Software Engineering}

Software development is based on software engineering, which is an engineering discipline that covers all aspects of software production. The goal is the cost-effective development of software systems where there are no physical limitations on the software's potential. software, which can sometimes be complex and difficult to understand [10]. Another concept about software engineering that could be defined as the establishment and application of engineering principles to obtain software. Taking into account factors as important as the economic cost, the reliability of the system and an efficient operation that meets the needs of the user [11].

\section{Waterfall Model}

The life cycle initially proposed by Royce in 1970 [12], shown in Fig. 1, was adapted for the software from the life cycles of other branches of engineering. It is the first of the proposed and most widely followed by organizations (it is estimated that $90 \%$ of the systems have been developed under this method) [11].

It works on the basis of documents, that is, the entry and exit of each phase is a specific document type deliverable. Ideally, each phase could be done by a different team thanks to the documentation generated between the phases. The documents are: Analysis: take as input a description in natural language of what the client wants. Produces the Software Requirements Document (SRD). Design: The entry is the SRD, produce the Software Design Document (SDD). Coding: From the SDD, produces modules. In this phase, unit tests are also carried out. Tests: The integration and testing of the entire system is carried out from the approved modules. The result of the tests is the final product ready to deliver [13].

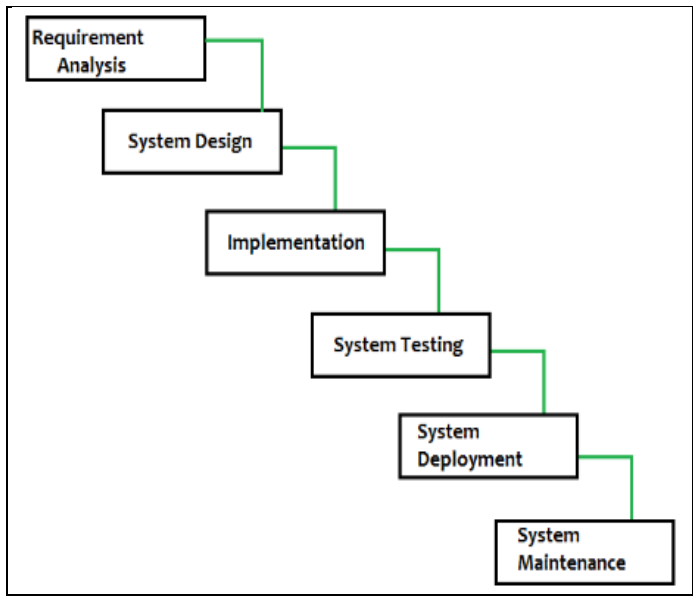

Fig. 1. Waterfall model

This model has some advantages as the planning is simple, the quality of the resulting product is high and it allows working with low-qualified personnel. By the other hand the disadvantages are the need to have all the requirements at the beginning. Typically, the client does not have perfectly 
defined system specifications, or unexpected needs may arise, if mistakes have been made in one phase it is difficult to go back, you do not have the product until the end, if an error is made in the analysis phase it is reflected until the delivery, with the consequent waste of resources. The client will not see results until the end.

\section{Software used for the Development of the Application}

Microsoft SQL Server: SQL Server is a Microsoft Relational Database Management System (RDBMS) that was designed for the business environment. SQL Server runs on TSQL (Transact-SQL), a set of Sybase and Microsoft programming extensions that add several features to standard SQL, including transaction control, exception and error handling, row processing, as well as declared variables. It also supports the management of capabilities for business intelligence and data mining [14].

Visual Studio 2015: It is an Integrated Development Environment (IDE) for Windows operating systems. Supports multiple programming languages, such as $\mathrm{C}++, \mathrm{C}$ \#, Visual Basic .NET, F \#, Java, Python, Ruby and PHP, as well as web development environments, such as ASP.NET MVC, Django, and others [15].

\section{METHODOLOGY}

The methodology phases were: Analysis of TMT components, requirements for the analysis, software specifications for application development, design and codification, testing and maintenance, and implementations and results. Fig. 2 displays the methodology phases.

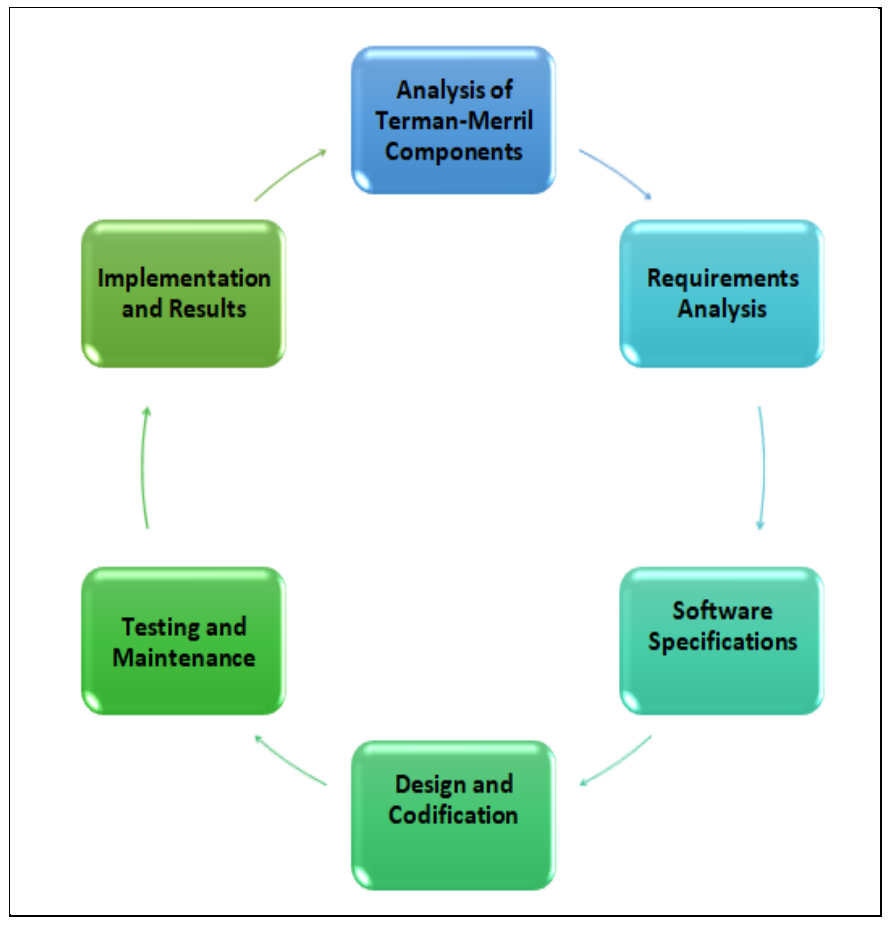

Fig. 2. Methodology phases.
Regarding the methodology to perform the research, first a detailed analysis of the requirements for the specifications of the ten series that make up the TMT was carried out, the application was developed complying with all the specifications regarding required data; how to evaluate each series, time needed, and results obtained.

The biggest challenge was the application development, since each series requires a different evaluation; the evaluation was based on a template with the correct answers and the final obtaining of the IQ; which is the data that the evaluator requests from the application.

Once the application was developed, it was applied in a sample of thirty students of the Bachelor's degree in Human Resources and in a company of the public transport branch to evaluate the applicants, obtaining successful results compared against other psychometric test such as Cleaver test.

Finally, it has been implemented in the human resources laboratory of the Faculty of Accounting and Administration and in the firm.

\section{A. Analysis Diagrams}

Some diagrams produced during analysis phase were displayed in Fig. 3 and 4. In Fig. 3 the application processes were shown.

The main requirements for the development in the first phase, users registry, a program where the general users data for registry in the application are entered, and with that, users can create their own account and password. After users registry phase was done, the instructions about the test were shown; before beginning the test series, samples of the questions were displayed. This process was done in the ten series of TMT. The responses were saved in the database; Fig. 4 shows part of the database diagram.

The result processing was activated through a buttom in the application once the series were responded. The TMT score obtained was calculated in the next way, first the series questions were saved in a table with the correct response; in other table related, the user answers were saved and a score was generated based in the answers and the correct answer. In this way, all the answers of the complete series were calculated and stored in a table named seriesUsers.

The user can see all the scores obtained in the ten series and visualize a result. The scores obtained place the user at a level from deficient, inferior, medium low, medium, medium high, superior to the outstanding level, which is the highest.

A part of the design of the database is shown in Fig. 4, where the main entities that are represented are: Users, SeriesUsers, Series, Questions, Answers, User Answers, among others.

With this scheme it is intended to solve the storage of the necessary data required by the TMT web application in all its processes. 


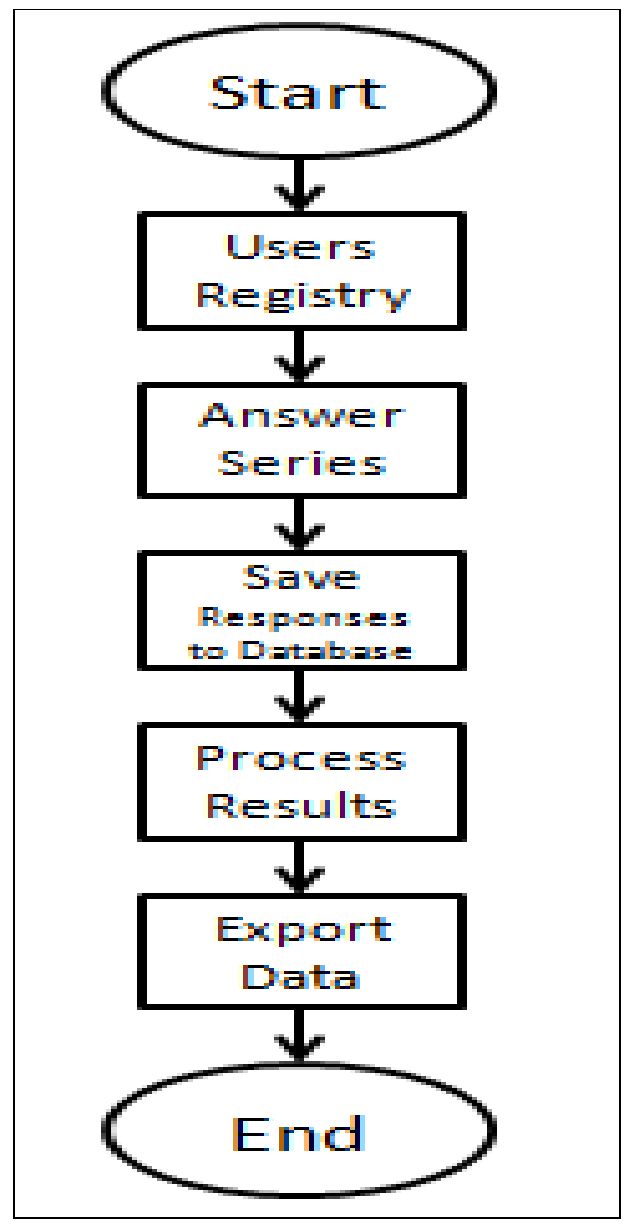

Fig. 3. Data flow processes.

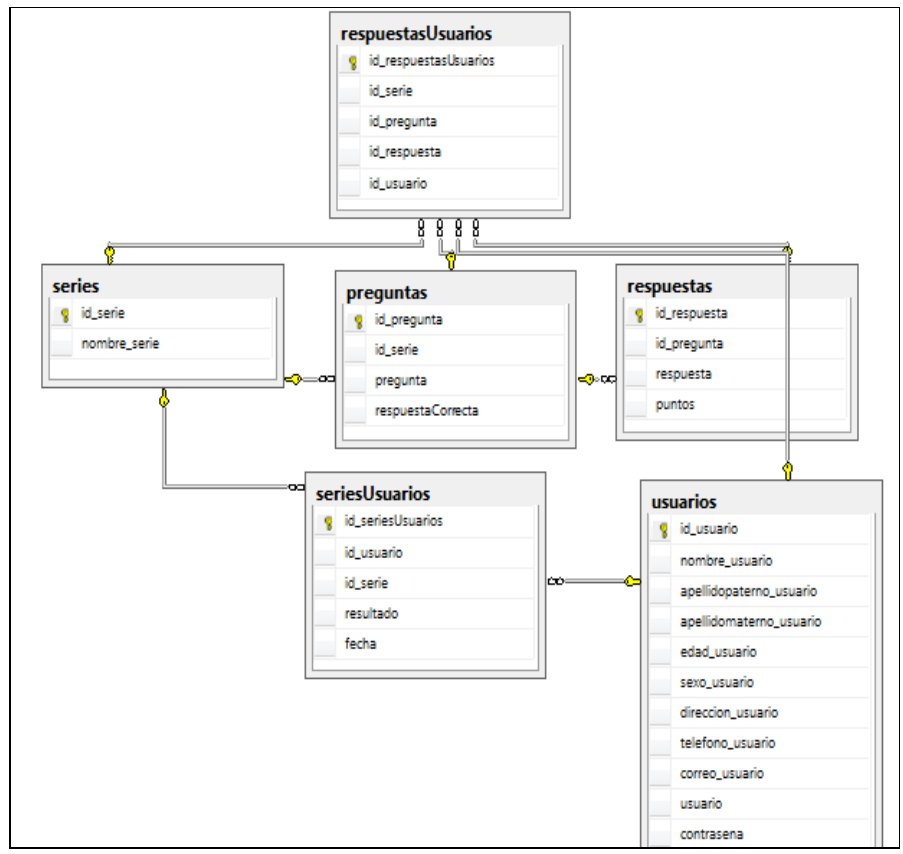

Fig. 4. Data model.

The User Registration Process is shown in Fig. 5, where the user's general data are recorded.

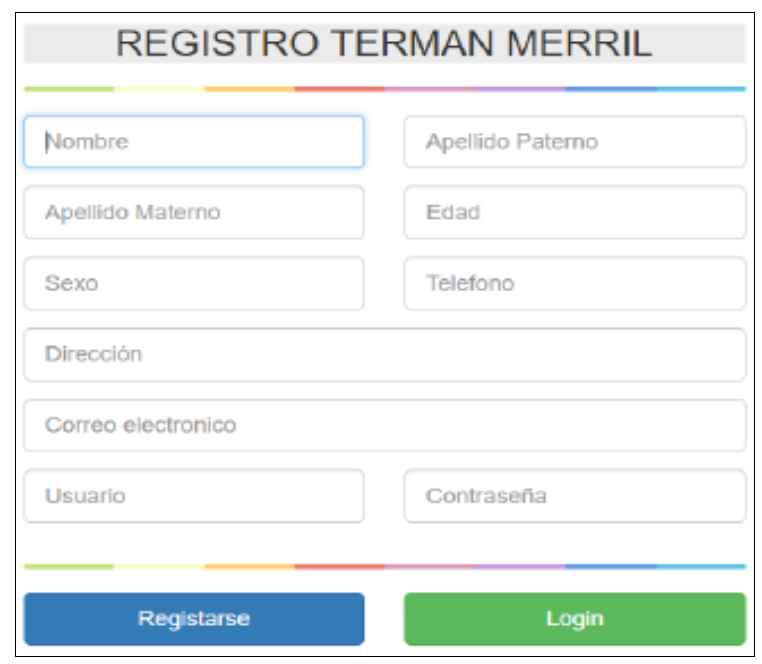

Fig. 5. Users registry.

A part of the program code that contains the user's registry is shown in Fig. 6.

Each of the series has its own programming since they have different questions, answers and way of evaluating.

The application was implemented in a university Windows server, the database recorded the responses to each of the series and this information can be exported to spreadsheets for been evaluated by human resources personnel.

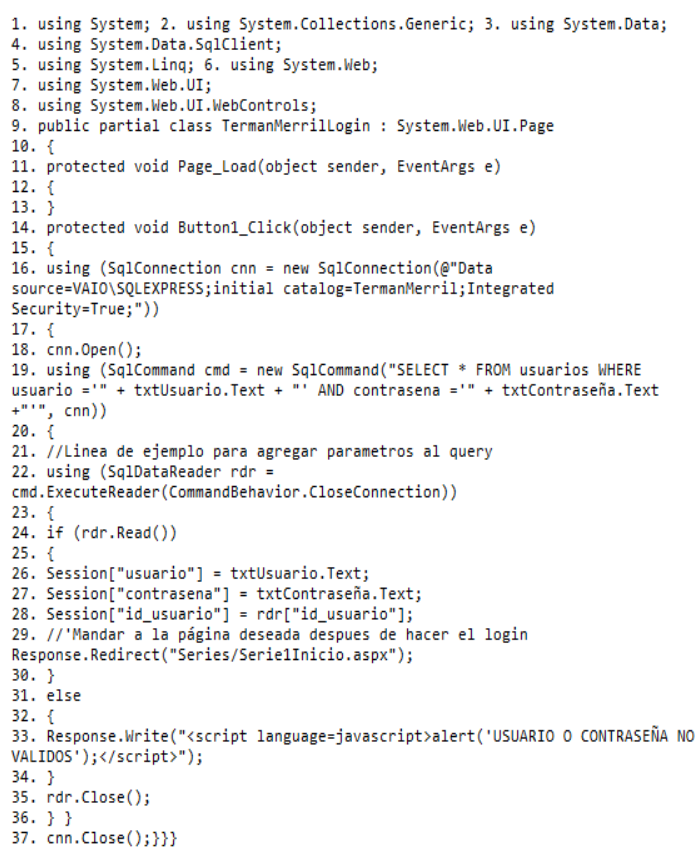

Fig. 6. Code for users registry.

\section{RESULTS}

Once the application has been implemented in the faculty repository, tests have been carried out with users-students of the Accounting and Administration faculty who are studying the Bachelor's degree in Human Resources, to whom the program is useful as a laboratory. 
It has also been used in the evaluation of a transport company where fifteen people were evaluated for hiring, reflecting a high degree of acceptance among the company's human resources managers. The application has a simple and effective design at the same time; since it provides the pertinent information about the people who answer it, so it can be implemented in any company or organization to evaluate the intellectual capacities of the users.

Fig. 7 shows the results of the application of the test in the company, where the data of ten people were shown, with age, the results of the ten tests and the IQ obtained.

The results obtained from the application of the test in the group of students, who answered it in the established time, where the system records the responses of each person and the intellectual coefficient obtained for each user.

These data are plotted and visualized by the test applicator.

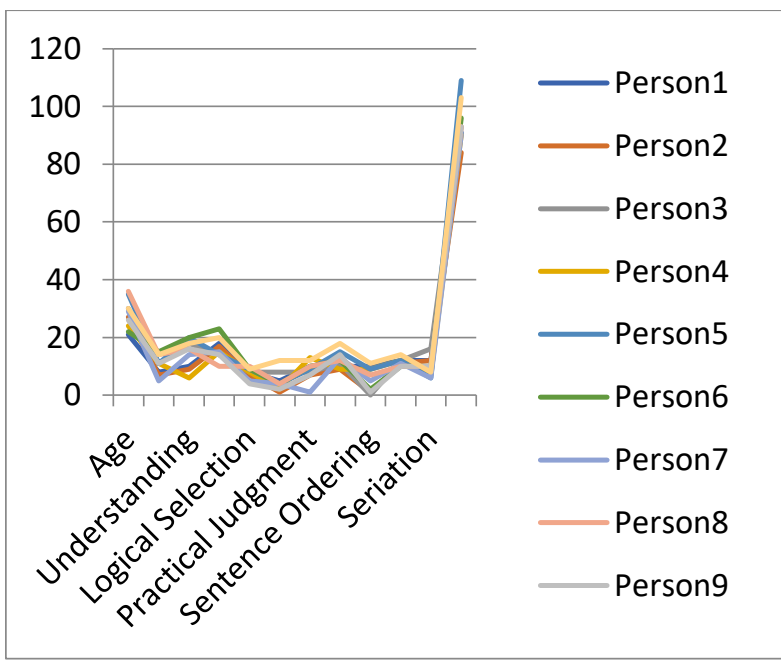

Fig. 7. Results of the Terman-Merril intelligence test application.

The application export the results to Excel spreadsheet for the graphics purposes, the application provides sufficient basis to make a decision to hire or not hire staff, other factors may influence the final decision of human resources personnel.

\section{CONCLUSION}

In this project, a web computational application has been designed and implemented for solving the needs of the human resources department at the Faculty of Accounting and Administration at Autonomous University of Coahuila, Mexico. Especially in the hiring of new personnel and else as an application that can be used for students in a computing laboratory, so can serve for various purposes as practices for the students and for uses in companies for evaluating new personnel to be hired.

The software has some modules: users' registry, answer series, process results, and export data, between others.

It should be noted that in the development of the TM application now plays an important role with students and teachers from Accounting Faculty and can be applied at human resources companies for helping. Some phases were defined in order to fulfillment the project; these were: Analysis of the TM components, requirements analysis, software specifications, design and codification, testing and maintenance, and implementation and results; using the waterfall model as a methodology.

The main results obtained were the process automation of the new personnel evaluation, made through a computational application, this implies better time response on the process, have the electronic information of the candidates for a position, less margin of error when applying calculations, and have a historical record of the candidates.

The implementation of the solution allowed appreciate how the use of IT, can support the processes of management and decisions in contexts such as university and companies, and contribute to strengthening initiatives aimed at improving decision-making process supported with the values obtained from the application.

The methodology used facilitated all the application development process.

\section{REFERENCES}

[1] O. Shcherbakova, D. Makarova, and E. Nikiforova, "Concept syhthesis test for intelligence research:qualitative analysis and conceptual commentaries". Bulletin of St. Petersburg State University. Psychology and Pedagogy, 2017, 2(7): p. 128-136..

[2] S. Ismat and J. Sagir, "A study of intelligence measure using Raven Standard Progressive Matrices Test items by principal components analysis" . Fuuast Journal of Biology, 2015. 5(1): p. 169-173.

[3] K. Grew and D. Flanagan, The intelligence test desk reference1998, U.S.A.: Boston7 Allyn and Bacon, pp. 65-90.

[4] M. Kholodnaya, and E. Volkova, "Conceptual Structures, Conceptual Abilities and Productivity of Cognitive Functioning: The Ontological Approach". Procedia-Social and Behavioral Sciences, 2016, 217(1): p. 914-922.

[5] N. Lemann, "The IQ Meritocracy". Time International, 1999. 153(12): p. 83-93.

[6] J. Ballesteros, "How the Terman scale measures intelligence. General psychometric model. Revised rules and updated results". Journal of Educational Psychology, 2011. 17(2): p. 179-193.Meyer, J., Le controle de gestion1969, Paris, France: Presses Universitarires.

[7] M. Amundsen and P. Litwin, Creating web sites with ASP.NET2007, España: Prentice Hall, pp. 45-67.

[8] A. Brust and S. Forte, Programación avanzada con Microsoft SQL Server 2005,2007, México, D.F.: McGraw-Hill, pp. 34-56.

[9] J. Ballesteros, "A critical review of the Terman scale. Why we should not use the Third Edition Stanford-Binet Form L-M". Psicología Educativa, 2010. 16(1): p. 23-30.

[10] I. Sommerville, Ingeniería del software.Séptima edición, ed. P. Educación2005, Madrid, España: Pearson educación. 712.

[11] R. Pressman, Ingeniería del software. Un enfoque práctico, ed. McGrawHill. Vol. 7a. Edición. 2010, México, D.F.: McGraw-Hill.

[12] W. Jedzej, "An approach to analysis and implementation from the waterfall model to the two-segmental model of information systems lifecycle". Business Informatics, 2012. 3(25): p. 195-206.

[13] R. Stephens, Beginning software engineering, 2015, Canada: Wiley Publishers, pp. 67-89.

[14] J. Gabillaud, Sql server 2016; aprender a administrar una base de datos transaccional con Sql server managament studio, 1a. ed., 2017, Madrid, 2017, Editorial ENI, pp. 215-234.

[15] S. Putier, Vb.Net y Visual studio 2015 los fundamentos del lenguaje, 1a. ed., 2017, Madrid :Editorial ENI, pp. 154-170. 